\title{
ACUTE INTERSTITIAL PNEUMONIA (HAMMAN-RICH SYNDROME) IN IDIOPATHIC PULMONARY FIBROSIS AND BRONCHOALVEOLAR CARCINOMA: A CASE REPORT
}

\author{
Jiri Plasek ${ }^{\mathrm{a} *}$, Jana Dvorackova ${ }^{\mathrm{b}}, \mathrm{Jan}_{\mathrm{Jahoda}}{ }^{\mathrm{c}}$, Kristina Trulikova ${ }^{\mathrm{d}}$, Radka Mokosova $^{\mathrm{e}}$, \\ Tomas Danek ${ }^{\mathrm{f}}$, Vladimir Hrabovsky ${ }^{\mathrm{a}}$, Arnost Martinek ${ }^{\mathrm{a}}$
}

\begin{abstract}
a Clinic of Internal Medicine, Faculty of Medicine, University of Ostrava and University Hospital Ostrava, Czech Republic
${ }^{b}$ Institute of Pathology, Faculty of Medicine, University of Ostrava and University Hospital Ostrava

c Clinic of Anaesthesiology and Resuscitation, Faculty of Medicine, University of Ostrava and University Hospital Ostrava

d Institute of Radiology, Faculty of Medicine, University of Ostrava and University Hospital Ostrava

e Clinic of Tuberculosis and Respiratory Diseases, Faculty of Medicine, University of Ostrava and University Hospital Ostrava

$f$ Cardiac Surgery Center, Faculty of Medicine, University of Ostrava and University Hospital Ostrava

E-mail:jiri_plasek@centrum.cz
\end{abstract}

Received: March 15, 2011; Accepted with revision: July 11, 2011; Available online: September 8, 2011

Key words: Acute Respiratory Failure/Interstitial Lung Disease/Adenocarcinoma

Aim. Acute interstitial pneumonia is characterized by rapid progressive dyspnoea degenerating into respiratory failure requiring mechanical ventilation. Acute interstitial pneumonia (AIP) and idiopathic pulmonary fibrosis (IPF) are separate clinic/pathological entities although overlap may be present. It is well-known that patients with IPF have increased risk of lung carcinoma; Adenocarcinoma in connection with IPF is less common. Moreover the subtype of adenocarcinoma, diffuse bronchoalveolar carcinoma has not yet been described.

Case report. We report the case of $45 \mathrm{yr}$ old former hockey player with increased bilateral reticular shadowing on chest radiograph, dyspnoea, velcro-like crackles, restrictive respiratory disease and mixed high-resolution computed tomography finding. During brief in-patient treatment the patient developed acute respiratory failure accompanied by multiorgan failure and disseminated coagulopathy. Deterioration of the microcirculation was followed by loss of peripheral vascular resistance, which was irreversible even with normalization of the blood gases achieved by extracorporeal membrane oxygenation. At autopsy, bronchoalveolar carcinoma in usual interstitial pneumonia (UIP) combined with areas of alveolar damage with hyaline membranes was found.

Conclusion. This case alerts clinicians to unusual idiopathic pulmonary fibrosis manifestations and its complications. Close collaboration between clinicians, pathologists and laboratory physicians is highly recommended for early diagnosis and appropriate treatment.

\section{INTRODUCTION}

Idiopathic pulmonary fibrosis (IPF) has been used as an umbrella term to describe different types of interstitial fibrotic lung diseases. In 1935, Hamman and Rich first described pulmonary fibrosis of unknown cause in 4 patients with acute diffuse interstitial fibrosis who died within 1-6 months of presentation ${ }^{1}$. Currently, although there is still a broad area of uncertainty, we recognize 7 types of fibrotic pulmonary disease of unknown aetiology, classified under the unifying concept of idiopathic interstitial pneumonia (IIP). IIP is defined ${ }^{2}$ as a heterogeneous group of damaged pleural parenchyma caused by different patterns of inflammation and fibrosis in contrast to alveolar airspace disease in bacterial pneumonia. Acute interstitial pneumonia (AIP) is a rare disease of lung interstitium, characterized by rapid onset and progression, with unfavorable prognosis and usually requiring mechanical ventilation $^{2}$. Since many clinical and pathological features of AIP are similar to those of adult respiratory distress syndrome, it can be difficult to make the distinction. However, the diagnosis of AIP should only be made if no known cause can be identified. The overall incidence of diffuse lung disease in a Danish study with a cohort of 21,765 patients showed a prevalence of $0.003 \%$ (ref. $^{3}$ ). Another study from the UK, reported the incidence of IPF to be $0.004 \%$ (ref. $^{4}$ ). The average length of survival from the time of diagnosis varies between 2.5 to 3.5 years $^{5}$. For AIP the incidence is not available but the prognosis is generally poor, most patients dying at initial disease manifestation $^{6}$. According to the ATS/ERS classification, for IPF, histological features of usual interstitial pneumonia (UIP) should be present, whereas in AIP diffuse alveolar damage is the most prominent pattern ${ }^{2}$. Friedrich was the first to use the concept of fibrosis associated lung cancer formerly called "scar cancer" in 1939 (ref. ${ }^{7}$ ). The largest sample of patients from the Mayo clinic with histologically proven UIP in the setting of IPF demonstrated that $67 \%$ had lung carcinoma and IPF diagnosed within four months of one another but only solid lesions with squamous cell carcinoma predominance was observed ${ }^{8}$. It must be stressed that mutual relationships exist, i.e. both patients with IPF are at increased risk of developing 
cancer and lung cancer can precede the diagnosis of IPF. However the latter is less frequent.

\section{CASE REPORT}

A 45 yr old Caucasian man, a former ice-hockey player, presented at the emergency department (ED) with moderate dyspnoea and inspiratory pain localized in the right hemithorax. He had an episode of joint swelling, febricity, proximal muscle weakness and unspecified fatigue which had lasted for a few days 3 weeks prior to ED admittance. For at least half a year, he had noticed gradual onset of post-exercise shortness of breath, which he had never experienced before, after his usual workload. The patient was a non-smoker with an unremarkable family history and was not on any medication. The only abnormality on a previous abdominal ultrasound was a fatty liver but no environmental or occupational risk was involved. Physical examination revealed the presence of velcro-like crackles in both basal lung fields posteriorly, an axial temperature of $37.2{ }^{\circ} \mathrm{C}$ and a mild decrease of peripheral oxygen saturation of $90 \%$ while breathing room air. The chest radiograph was near normal with mild basal predominantly right reticular shadowing (Fig. 1A). For laboratory values at admissison and after 2 weeks as inpatient see (Table 1). Laboratory findings on admission included leukocytosis with prevalent neutrophilia, mild elevation of C-reactive protein and strikingly elevated liver enzymes; also creatinkinase and myoglobin were recognized.

This was interpreted as possible right basal pneumonia and myositis of unknown aetiology. Saline induced sputum tested negative both for bacteria and fungi. A full panel of respiratory viruses serology analysis proved noncontributory. Antibiotics and corticoid pulse therapy to a cumulative dose of $3 \mathrm{~g}$ of Solumedrol were administered. Meanwhile, mild positivity of anti-Ro-52 (Western blot) antibody and ELISA IgM rheumatoid factor (127.37 IU/ $\mathrm{ml}$ ) were determined, and during 2 weeks of in-patient therapy good response was obtained both as decreased levels of WBC, CK, liver enzymes and clinically subsid-
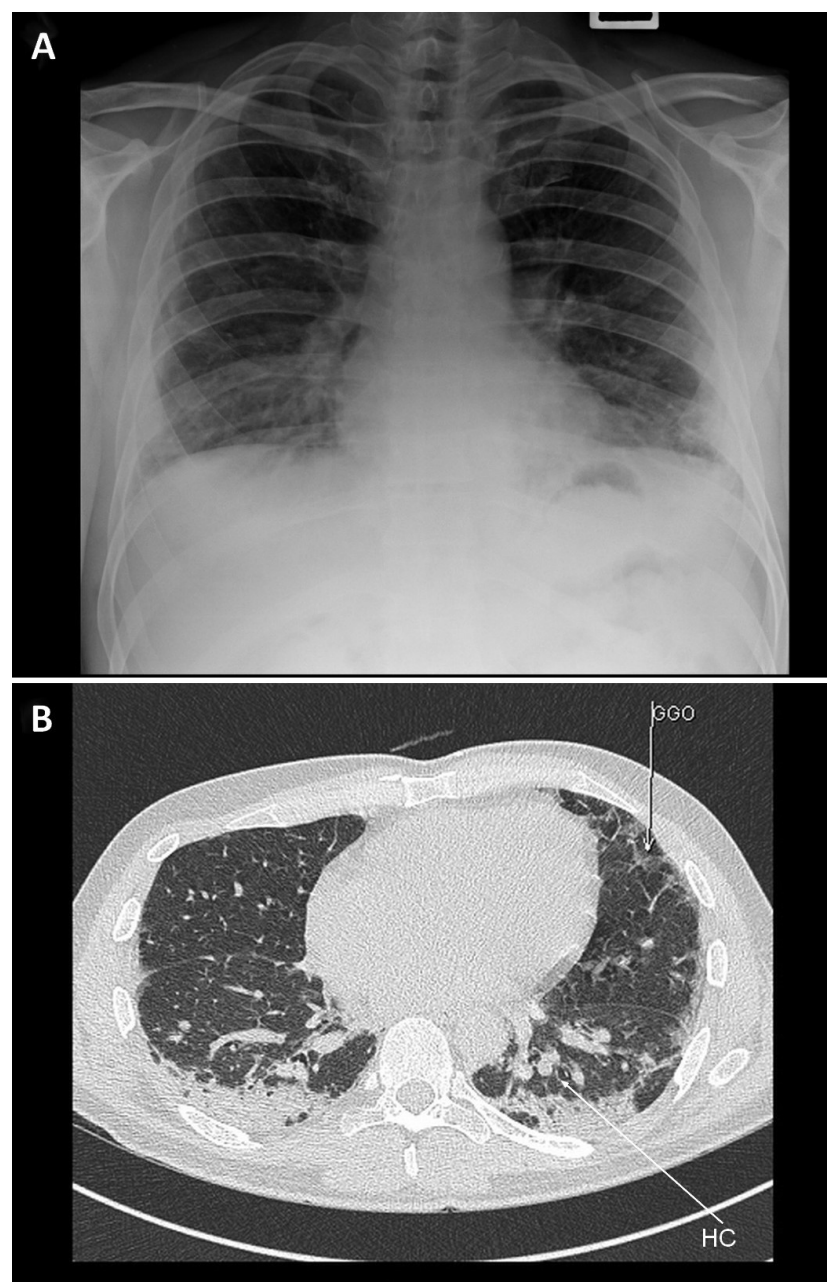

Fig. 1. A) Chest radiograph shows near normal appearance with mild basal bilateral reticular shadowing, predominantly right $\mathbf{B}$ ) Chest high-resolution computed tomography shows bilateral basal patchy consolidation with scarce honeycombing (HC), interlobular and intralobular septal thickening and ground glass opacities (GGO) anteriorly.

Table 1. Comparison of selected biochemical parameters over 2 weeks of in-patient care.

\begin{tabular}{|c|c|c|c|}
\hline & & At Admission & After 2 weeks as in-patient \\
\hline White blood cell count & & $19 * 10^{9}$ & $13 * 10^{9}$ \\
\hline Creatinkinas & $(\mu \mathrm{kat} / 1)$ & 61.4 & 5.1 \\
\hline Myoglobin & $(\mu \mathrm{g} / \mathrm{ml})$ & 623 & 86 \\
\hline C-reactive protein & $(\mathrm{mg} / 1)$ & 17 & 14 \\
\hline Alaninaminotransferase & $(\mu \mathrm{kat} / 1)$ & 8.2 & 4.9 \\
\hline Aspartataminotransferase & $(\mu \mathrm{kat} / 1)$ & 17.6 & 5.7 \\
\hline Alcalic phosphatise & $(\mu \mathrm{kat} / 1)$ & 1.9 & 1.5 \\
\hline$\gamma$-glutamyl transferase & $(\mu \mathrm{kat} / 1)$ & 1.2 & 3.2 \\
\hline
\end{tabular}



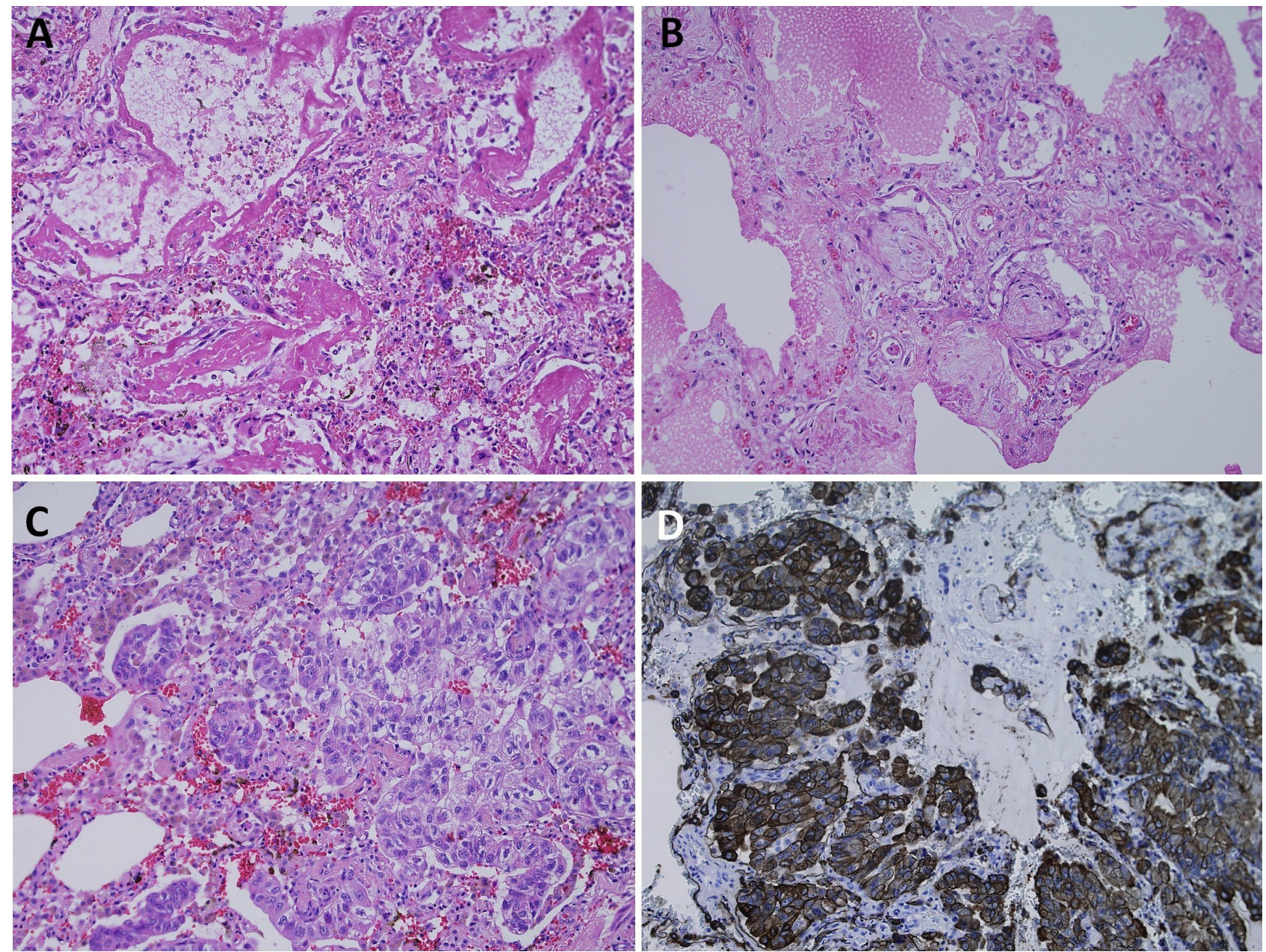

Fig. 2. Histological demonstration of lung tissue. A) with features of diffuse alveolar damage, hyaline membranes, part of the alveolar septa with fibroblastic proliferation and patchy alveolar septal infiltrates of mononuclear cells B) with fibroblastic foci, usual interstitial pneumonia pattern, spindle-shaped cells in oedematous stroma C) with locus of bronchoalveolar carcinoma $\mathbf{D}$ ) with locus of bronchoalveolar carcinoma in fibrotic terrain labelled immunohistochemically for cytokeratin 7 as marker of malignant proliferation. Haematoxylin-eosin staining, 200x magnification, Olympus DP70.

ing fatigue and muscle weakness, respectively. No other autoimmune markers (ANCA, anti-keratin, autoimmune hepatitis screening, anti-Ds-DNA) were found to be positive. Electromyography showed signs of a diffuse myopathic pattern, while no inflammatory infiltration was identified from muscle biopsy. Restrictive respiratory disease pattern at spirometry $(\mathrm{VC} \max =1.21, \mathrm{FEV} 1=1.11$, Tiffenau index $=0.95$ ) was identified. High resolution CT scan demonstrated basal and posterior pleural thickening, reticular shadowing and both ground glass opacities and honeycombing aligned (Fig.1B). Treatment began with Azathioprine 50mg/day, Prednisone

$1 \mathrm{mg} / \mathrm{kg}$ and $3 \times 600 \mathrm{mg}$ of acetylcystein daily as a treatment for suspected IPF where 2 major and 2 minor ATS/ERS criteria for IPF were satisfied. A consecutive bronchoscopy revealed a granulocyte count $(65 \%)$ and imunoregulatory index (CD4/8) of 0.85 , and atypical cells in the cytology; however the samples could have been influenced by corticosteroid therapy. During one week, rapid worsening of the global clinical status of the pa- tient including pyrexia, fatigue recurrence and increased dyspnoea were observed.

Since pulse oxymetric oxygen saturation dropped below $85 \%$, non-invasive ventilation was instituted (pressure support $15 \mathrm{~cm} \mathrm{H}_{2} \mathrm{O}$, positive end expiratory pressure $6 \mathrm{~cm}$ $\mathrm{H}_{2} \mathrm{O}$, Inspiratory $\mathrm{O}_{2}$ fraction $90 \%$ ). Since $7 \mathrm{~h}$ of non-invasive ventilation brought no optimal tissue oxygenation, invasive ventilation followed in this mode: P-SIMV (pressure synchronized mandatory ventilation), inverse proportion of inspiration to expiration ratio 2:1 in advance of inspiration, tidal volume $5-8 \mathrm{ml} / \mathrm{kg}$ and PEEP of $8-10 \mathrm{~cm} \mathrm{H}_{2} \mathrm{O}$.

After $26 \mathrm{~h}$ of lung-protective ventilation, we observed a clear and rapid trend to global respiratory failure with the collapse of adequate respiratory gas exchange. Due to unfavourable blood gas developments, we decided to establish veno-venous exctracorporeal membrane oxygenation (ECMO) circuit via right internal jugular vein and right femoral vein. Centrifugal pump (Biomedicus 550 Bio-Pump Medtronic, Inc., USA) and oxygenator (Hilite LT 7000, rheoparin coated, Medos Medizintechnik AG, Germany) were used. 
Initial settings were $3500 \mathrm{rpm}$, with blood flow from 3,5 $\mathrm{lpm}$ to $4,5 \mathrm{lpm}$, gas flow through the gas blender 8 $1 \mathrm{pm}$ with oxygen fraction 1.0. Ventilator settings were: tidal volume $5 \mathrm{ml} / \mathrm{kg}$, oxygen fraction 0.5 , pressure plateau $25 \mathrm{~cm} \mathrm{H} \mathrm{H}_{2} \mathrm{O}$, PEEP $10 \mathrm{~cm} \mathrm{H_{2 }} \mathrm{O}$. With ECMO normalization of the blood gases while keeping oxygen saturation above $96 \%$ was reached.

Despite comprehensive critical care, the patient died of multi-organ failure in a few hours after ECMO initiation, which was only 19 days after initial presentation. The autopsy revealed not only features of usual interstitial pneumonia (UIP) but also diffuse growing multilocular bronchoalveolar carcinoma and areas of diffuse alveolar damage (DAD) with typical hyaline membranes (Fig. 2).

\section{DISCUSSION}

This study presents a case of a well-differentiated subtype of adenocarcinoma namely bronchoalveolar carcinoma (BAC) in idiopathic pulmonary fibrosis complicated by acute interstitial pneumonia (AIP, formerly Hamman-Rich syndrome) diagnosed at the same time and developing into fatal respiratory and subsequently multi-organ failure.

While the association of lung cancer and IPF has been extensively studied and is well established in the current medical literature, no BAC that demonstrates diffuse growth has been highlighted ${ }^{6-8}$. Moreover the Mayo clinic register shows that squamous cell carcinoma outnumbers adenocarcinoma in IPF ( ref. $^{8}$ ). The diagnosis of IPF according to ATS/ERS was finally based on 2 major criteria; (abnormal pulmonary function and impaired gas exchange, bibasilar reticular abnormalities on HRCT), 2 minor criteria (Velcro-like crackles, insidious onset of otherwise unexplained dyspnoea on exertion) and histological confirmation of UIP, where key features are satisfied (Fibroblastic foci at the edge of a scar, patchy lung involvement with sub pleural distribution). In addition, no occupational or environmental risk was present, idiopathic inflammatory myopathia per se was not confirmed on muscle biopsy, neither eosinophilia nor hypercalcaemia was found, and serum and sputum screening for tuberculosis and other bacterial, fungal or viral infection proved negative. The radiological appearance was mixed, employing both ground glass opacities in some areas and honeycombing in others, which was located mostly to the posterior basal sub pleural parts of the lungs. Nonetheless, the histological confirmation was challenging as both features of UIP were present in some areas and DAD in others.

According to the clinical presentation, characterised by gradual onset of exercise dyspnoea followed by rapid dyspnoea exacerbation with febricity observed together with combined histological features (UIP, DAD), the diagnosis of AIP superimposed on IPF was established. In addition, the presence of BAC supports the premise of a longer lasting fibrotic process. In this way, it poses the question whether the present classification of interstitial lung disease is comprehensive enough to cover all the disease manifestations.

The type of carcinoma described here probably originates in bronchiolar cells; the pure histological pattern in lung adenocarcinoma is quite rare, however ${ }^{9}$. BAC is described as a tumor without stromal, pleural or vascular invasion $^{10}$. There is no doubt that the trio of $\mathrm{Th}_{2}$ lymphocyte inflammatory response, repeated acute parenchymal damage and aberrant healing is the cornerstone of IPF, yet the stimulus initiating this process has not been identified $^{11}$. Increased neutrophile bronchoalveolar lavage level and serum IL-8 portends disease activity in IPF (ref. ${ }^{12}$ ). Alternatively, the degree of neutrophil infiltration in BAC is associated with poor prognosis ${ }^{13}$. Although not the initiator of either IPF or BAC pathogenesis, the number of neutrophilic elements in the lesion and increased oxidative stress may indicate a possible link between IPF and BAC. Elevated creatine kinase and myoglobin plasma level has been reported in lung cancer as a marker of disease manifestation ${ }^{14-16}$. Although not specific only for lung cancer, it might be useful to implement this easy and cheap blood sampling in IPF evaluation as an additional screening method for possible lung cancer.

\section{CONCLUSION}

The major contribution of our case report we see in: 1) presentation of an unique case with coincidence of AIP, IPF and BAC pointing to the link between lung cancer and fibrotic pulmonary disease 2) drawing attention to how the manifestation of lung cancer might overlap the appearance of IPF, AIP 3) understanding of the earlier onset of lung cancer in IPF patients 4) identifying diffuse growing adenocarcinoma as opposed to typical solid lesions 5) demonstrating AIP as a fatal form of IPF exacerbation. 6) identifying myopathy (elevated creatine kinase or myoglobin) as a paraneoplastic sign of lung cancer that might be used as a marker of disease manifestation. In conclusion, this case reported should alert clinicians to an unusual IPF manifestation and complications with poor outcome in a young, at referral oligosymptomatic patient, where BAC was latent due to AIP+IPF clinicalmorphological appearance. Close collaboration between clinicians, laboratory physicians and pathologists with emphasis on pertinent clinical presentation and early laboratory marker evaluation should be encouraged in the setting of IPF investigation.

\section{ACKNOWLEDGEMENT}

The present authors would like to thank Barbora Blahova and Eva Maria O'Reilly for their writing assistance.

There is no conflict of interest related to present article.

Author's contribution: JP was primary treating physician, initiator and main manuscript contributor, who is responsible for the integrity of the work as a whole. JD is responsible for the whole histopathological content including microscopic 
pictures and autopsy. JJ was treating physician and is responsible for description of ventilatory support. KT is responsible for radiological description including $x$-ray and CT scan. $R M$ was treating physician and is responsible for respiratory disease classifications. TD is responsible for extracorporeal membrane oxygenation settings description. VH and AM are senior-authors, who reviewed the work and made substantial changes in the final work.

\section{REFERENCES}

1. Hamman L, Rich AR. Fulminating diffuse interstitial fibrosis of the lungs. Trans Am Clin Climatol Assoc 1935;51:154-63.

2. American Thoracic Society/European Respiratory Society International Multidisciplinary Consensus Classification of the Idiopathic Interstitial Pneumonias. This joint statement of the American Thoracic Society (ATS), and the European Respiratory Society (ERS) was adopted by the ATS board of directors, June 2001 and by the ERS Executive Committee, June 2001. Am J Respir Crit Care Med 2002; 165(2):277-304.

3. Kornum JB, Christensen S, Grijota M, Pedersen L, Wogelius P, Beiderbeck A, Sørensen HT. The incidence of interstitial lung disease 1995-2005: a Danish nationwide population-based study. BMC Pulm Med 2008;8:24

4. Gribbin J, Hubbard RB, Le Jeune I, Smith CJP, West J, Tata LJ. Incidence and mortality of idiopathic pulmonary fibrosis and sarcoidosis in the UK. Thorax 2006;61:980-5

5. King TE Jr, Costabel U, Cordier J-F, doPico GA, du Bois RM, Lynch D, Lynch JP III, Myers JL, Panos RJ, Raghu G, et al. Idiopathic pulmonary fibrosis: diagnosis and treatment. Am J Respir Crit Care Med 2000;161:646-64.
6. Bonaccirsi A, Cancellieri, Chilosi M, Trisolini R, Boaron M, Crimi N, Poletti. Acute interstitial pneumonia: report of a series. Eur Respir J 2003;21:187-91

7. Meyer EC, Liebow AA. Relationship of interstitial pneumonia honeycombing and atypical epithelial proliferation to cancer of the lung. Cancer 1965;18:322-51.

8. Aubry MC, Myers JL, Douglas WW, Tazelaar HD, Washington Stephens TL, Hartman TE, Deschamps C, Pankratz VS. Primary pulmonary carcinoma in patients with idiopathic pulmonary fibrosis. Mayo Clin Proc 2002; 77(8):763-70.

9. Hubbard, R., A. Venn, S. Lewis, J. Britton. 1999. Lung cancer and cryptogenic fibrosing alveolitis: a population based cohort study. Am J Respir Crit Care Med 161:5-8.

10. Brombilla E, Travis WD, Colby TW, Corrin B, Shimosato Y. The New World Health organisation classification of lung tumors. Eur Respir J 2001; 18: 1059-68.

11. Gross TJ, Hunninghake GW. Idiopathic pulmonary fibrosis. N Engl J Med 2001;7:517-25.

12. Ziegenhagen MW, Zabel P, Zissel G, Schlaak M, Müller-Quernheim J. Serum level of interleukin 8 is elevated in idiopathic pulmonary fibrosis and indicates disease activity. Am J Respir Crit Care Med 1998;3:762-8.

13. Raz DJ, He B, Rosell R, Jablons DM. Current Concepts in Bronchioloalveolar Carcinoma Biology. Clin Cancer Res 2006;12:3698-3704.

14. Bor MV. Interpretation of serum CK-MB activity measured by immunoinhibition assay requires special care in patients with neoplastic pathology. Int J Clin Lab Res 1999;29(3):133-4.

15. Wang HC, Lu JY, Ting YM. Elevated serum CK-MB and CK-BB isoenzymes in a patient with small cell lung cancer: a case report. Zhonghua Yi Xue Za Zhi 1995;55(3):270-3.

16. Flonta SE, Arena S, Pisacane A, Michieli P, Bardelli A. Expression and functional regulation of myoglobin in epithelial cancers. Am J Pathol. 2009;175(1):201-6. 
